\title{
XLVIII. Experimental researches in electricity.-Eleventh series
}

\section{Michael Faraday Esq. D.C.L. F.R.S.}

To cite this article: Michael Faraday Esq. D.C.L. F.R.S. (1838) XLVIII. Experimental researches in electricity.-Eleventh series, Philosophical Magazine Series 3, 13:83, 355-367, DOI: $10.1080 / 14786443808649595$

To link to this article: http://dx.doi.org/10.1080/14786443808649595

册 Published online: 01 Jun 2009.

Submit your article to this journal $\lceil\pi$

Џ Article views: 7

Q View related articles $\square$

Citing articles: 1 View citing articles 5 
Mr. Faraday's Experimental Researches in Electricity. 355

Had it been an ore of iron, reduced by a blast of lightning, we should not have found it alloyed with nickel.

We are therefore led to conclude, that our specimen is of celestial origin, and that it is a fragment of one of those asteroids of cometary matter, which wandering in space, occasionally cross our orbit, and being attracted by the earth, so that they rush through our atmosphere, bursting into fire and descending, take up their abode on this sublunary sphere.

Boston, May 29, 1838.

XLVIII. Experimental Rescarches in Electricity.-Eleventh Series. By Michaer. Faraday, Esq., D.C.L.F.R.S. Fullerian Prof. Chem. Royal Institution, Corr. Memb. Royal and Imp. Acadd. of Sciences, Paris, Petersburgh, Florence, Copenhagen, Berlin, \&c. \&c.

[Continued from p.299.]

\section{$\uparrow$ iv. Induction in curved Lines.}

1215. A MONGST those results deduced from the mole$A$ cular view of induction (1166.), which, being of a peculiar nature, are the best tests of the truth or error of the theory, the expected action in curved lines is, I think, the most important at present; for, if shown to take place in an unexceptionable manner, 1 do not see how the old theory of action at a distance and in straight lines can stand, or how the conclusion that ordinary induction is an action of contiguous particles can be resisted.

1216. There are many forms of old experiments which might be quoted as favourable to, and consistent with the view I have adopted. Such are most cases of electro-chemical decomposition, electrical brushes, auras, sparks, \&c.; but as these might be considered equivocal evidence, inasmuch as they include a current and discharge, (though they have long been to me indications of prior molecular action (1230.)) $I$ endeavoured to devise such experiments for first proofs as should not include transfer, but relate altogether to the pure simple inductive action of statical electricity.

1217. It was also of importance to make these experiments in the simplest possible manner, using not more than one insulating medium or dielectric at a time, lest differences of slow conduction should produce effects which might erroneously be supposed to result from induction in curved lines. 


\section{Mr. Faraday's Researches in Electricity. (Series XI.)}

It will be unnecessary to describe the steps of the investigation minutely; I will at once proceed to the simplest mode of proving the facts, first in air and then in other insulating media.

1218. A cylinder of solid shell-lac, 0.9 of an inch in diameter and seven inches in length, was fixed upright in a wooden foot (fig. 3.): it was made concave or cupped at its upper extremity so that a brass ball or other small arrangement could stand upon it. The upper half of the stem having been excited negatively by friction with warm flannel, a brass ball, $B, 1$ inch in diameter, was placed on the top, and then the whole arrangement examined by the carrier ball and Coulomb's electrometer $(1180.8 \mathrm{c}$.). For this purpose the balls of the electrometer were charged positively to about $360^{\circ}$, and then the carrier being applied to various parts of the ball $\mathrm{B}$, the two were uninsulated whilst in contact or in position, then insulated $*$, separated, and the charge of the carrier examined as to its nature and force. Its electricity was always positive, and its force at the different positions $a, b, c, d, \& c$. (fig. 3. and 4.) observed in succession, was as follows:

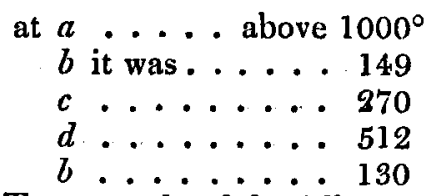

1219. To comprehend the full force of these results, it must first be understood, that all the charges of the ball $\mathrm{B}$ and the carrier are charges by induction, from the action of the excited surface of the shell-lac cylinder; for whatever electricity the ball $B$ received by communication from the shell-lac,either in the first instance or afterwards, was removed by the uninsulating contacts, only that due to induction remaining; and this is shown by the charges taken from the ball in this its uninsulated state being always positive, or of the contrary character to the electricity of the shell-lac. In the next place the charges at $a, c$, and $d$ were of such a nature as

* It can hardly be necessary for me to say here, that whatever general state the carrier ball acquired in any place where it was uninsulated and then insulated, it retained on removal from that place, notwithstanding that it might pass through other places, that would have given to it, if uninsulated, a different condition. 
might be expected from an inductive action in straight lines, but that obtained at $b$ is not so: it is clearly a charge by induction, but induction in a curved line; for the carrier ball whilst applied to $b$, and after its removal to a distance of six inches or more from $B$, could not, in consequence of the size of $\mathrm{B}$, be connected by a straight line with any part of the excited and inducing shell-lac.

1220. To suppose that the upper part of the uninsulated ball $B$, should in some way be retained in an electrified state by that portion of the surface which is in sight of the shell-lac, would be in opposition to what we know already of the subject. Electricity is retained upon the surface of conductors only by induction (1178.); and though some persons may not be prepared as yet to admit this with respect to insulated conductors, all will as regards uninsulated conductors like the ball $\mathrm{B}$; and to decide the matter we have only to place the carrier ball at $e$ (fig. 4.), so that it shall not come in contact with $\mathrm{B}$, uninsulate it by a metallic rod descending perpendicularly, insulate it, remove it, and examine its state: it will be found charged with the same kind of electricity as, and even to a higher degree Fig. 4.

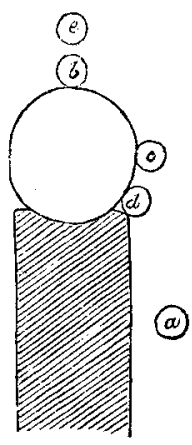
(12y4.) than, if it had been in contact with the summit of $\mathrm{B}$.

1221. To suppose, again, that induction acts in some way through or across the metal of the ball, is negatived by the simplest considerations; but a fact in proof will be better. If instead of the ball $B$ a small disc of metal be used, the carrier may be charged at, or above the middle of its upper surface; but if the plate be enlarged to about $1 \frac{1}{x}$ or 2 inches in diameter, $\mathbf{C}$ (fig. 5.), then no charge will be given to the carrier at $f$, though when applied nearer to the edge at $g$, or even above the middle at $h$, a charge will be obtained; and this is true thongh the plate may be a mere thin film of goldFig 5.

(3)

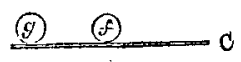
leaf. Hence it is clear that the induction is not through the metal, but through the air or dielectric, and that in curved lines.

1222. I had another arrangement, in which a wire passing downwards through the middle of the shell-lac cylinder to the earth, was connected with the ball B (fig. 6.) so as to keep it 


\section{Mr. Faraday's Researches in Electricity. (Series XI.)}

in a constantly uninsulated state. This was a very convenient form of apparatus, and the results with it were the same as those described.

1223. In another case the ball B was supported by a shell-lac stem, independently of the excited cylinder of shell-lac, and at half an inch distance from it; but the effects were the same. Then the brass ball of a charged Leyden jar was used in place of the excited shell-lac to produce induction; but this caused no alteration of the phænomena. Both positive and negative inducing charges were tried with the same general results. Finally, the arrangement was inverted in the air for the Fig. 6. purpose of removing every possible objection to the conclusions, but they came out exactly the same.

1224. Some results obtained with a brass hemisphere instead of the ball $\mathrm{B}$ were exceedingly interesting. It was 1.36 of an inch in diameter, (fig. 7.), and being placed on the top of the excited shell-lac cylinder, the carrier ball was applied, as in the former experiments (1218.), at the respective positions delineated in the figure. At $i$ the force was $112^{\circ}$, at $k 108^{\circ}$, at $l 65^{\circ}$, at $m 35^{\circ}$; the inductive force gradually diminishing, as might have been expected, to this point. But on raising the carrier to the position $n$ the charge increased to $87^{\circ}$; and on raising it still higher to $o$, the charge still further increased to $105^{\circ}$ : at a higher point still, $p$, the charge taken was smaller in amount, being $98^{\circ}$, and continued to diminish for more elevated positions. Here the induction fairly

Fig. 7.

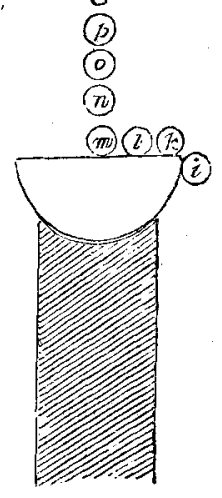
turned a corner. Nothing, in fact, can better show both the curved lines or courses of the inductive action, disturbed as they are from their rectilineal form by the shape, position, and condition of the metallic hemisphere; and also a lateral tension, so to speak, of these lines on one another: all depending, as I conceive, on induction being an action of the contiguous particles of the dielectric thrown into a state of polarity and tension, and mutually related by their forces in all directions.

1225. As another proof that the whole of these actions were inductive, I may state a result which was exactly what might be expected, namely, that if uninsulated conducting matter was brought round and near to the excited shell-lac stem, then the inductive force was directed towards it, and 
could not be found on the top of the hemisphere. Removing this matter the lines of force resumed their former direction. The experiment affords proofs of the lateral tension of these lines, and supplies a warning to remove such matter in repeating the above investigation.

1226. After these results on curved inductive action in air I extended the experiments to other gases, using first carbonic acid and then hydrogen: the phænomena were precisely those already described. In these experiments I found that if the gases were confined in vessels they required to be very large, for whether of glass or earthenware, the conducting power of such materials is so great that the induction of the excited shell-lac cylinder towards them is as much is if they were metal; and if the vessels be small, so great a portion of the inductive force is determined towards them that the lateral tension or mutual repulsion of the lines of force before spoken of, (1224.) by which their inflexion is caused, is so much relieved in other directions, that no inductive charge will be given to the carrier ball in the positions $k, l, m, n, o, p$, (fig. 7.). $A$ very good mode of making the experiment is to let large currents of the gases ascend or descend through the air, and carry on the experiments in these currents.

1227. These experiments were then varied by the substitution of a liquid dielectric, namely, oil of turpentine, in place of air and gases. A dish of thin glass well covered with a film of shell-lac, (1272.) and found by trial to insulate well, had some highly rectified oil of turpentine put into it to the depth of half an inch, and being then placed upon the top of the brass hemisphere, (fig. 7.) observations were made with the carrier ball as before (1224.). The results were the same, and the circumstance of some of the positions being within the fluid and some without, made no sensible difference.

1228. Lastly, I used a few solid dielectrics for the same purpose, and with the same results. These were shell-lac, sulphur, fused and cast borate of lead, flint glass well covered with a film of lac, and spermaceti. The following was the form of experiment with sulphur, and all were of the same kind. A square plate of the substance, two inches in extent and 0.6 of an inch in thickness, was cast with a small hole or depression in the middle of one surface to receive the carrier ball. This was placed upon the surface of the metal hemisphere (fig. 9.) arranged on the excited lac as in former cases, and observations were made at $n, o, p$, and $q$. Great care was required in these experiments to free the sulphur or other solid substance from any charge it might previously have received. This was done by breathing and wiping (1203.), 
360 Mr. Faraday's Researches in Electricity. (Series XI.)

and the substance being found free from all electrical excitement, was then used in the experiment; after which it was removed and again examined, to ascertain that it had received no charge, but had acted really as a dielectric. With all these precautions the results were the same: and it is thus very satisfactory to obtain the curved inductive action through solid bodies, as any possible effect from the translation of charged particles in fluids or gases, which some persons might imagine to be the case, is here entirely negatived.

1229. In these experiments with solid dielectrics, the degree of charge, as-

Fig. 9.

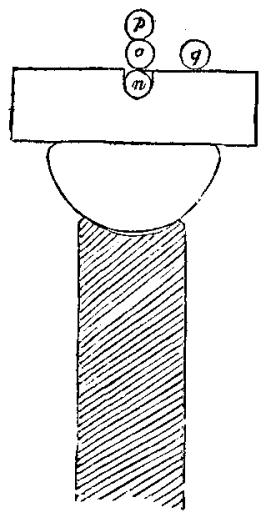
sumed by the carrier ball at the situations $n, o, p$ (fig. 9.), was decidedly greater than that given to the ball at the same places when air only intervened between it and the metal hemisphere. This effect is consistent with what will hereafter be found to be the respective relations of these bodies, as to their power of facilitating induction through them (1269. 1273. 1277.).

1230. I might quote many other forms of experiment, some old and some new, in which induction in curved or contorted lines takes place, but think it unnecessary after the preceding results; I shall therefore mention but two. If a conductor A, (fig. 8.) be electrified, and an uninsulated metallic ball $B$,

Fig. 8.

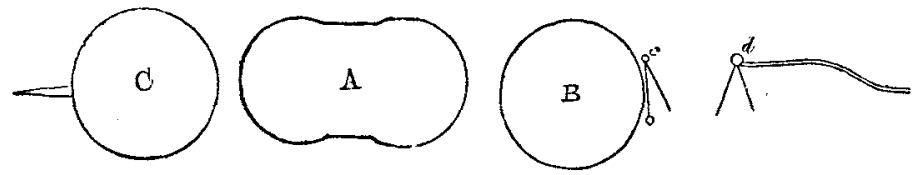

or even a plate, provided the edges be not too thin, be held before it, a small electrometer at $c$ or at $d$, uninsulated, will give signs of electricity, opposite in its nature to that of $\mathrm{A}$, and therefore caused by induction, although the influencing and influenced bodies cannot be joined by a right line passing through the air. Or if, the electrometers being removed, a point be fixed at the back of the ball in its uninsulated state as at $\mathrm{C}$, this point will become luminous and discharge the conductor A. The latter experiment is described by Nicholson*, who, however, reasons erroneously upon it. As to its

\footnotetext{
* Encyclopadia Britannica, vol. vi. p. 504.
} 
introduction here, though it is a case of discharge, the discharge is preceded by induction, and that induction must be in curved lines.

1231. As argument against the received theory of induction and in favour of that which $I$ have ventured to put forth, I cannot see how the preceding results can be avoided. The effects are clearly inductive effects produced by electricity, not in currents but in its statical state, and this induction is exerted in lines of force which, though in many experiments they may be straight, are here curved more or less according to circumstances. I use the term line of inductive force merely as a temporary conventional mode of expressing the direction of the power in cases of induction; and in the experiments with the hemisphere (1224.), it is curious to see how, when certain lines have terminated on the under surface and edge of the metal, those which were before lateral to them expand and open out from each other, some bending round and terminating their action on the upper surface of the hemisphere, and others meeting, as it were, above in their progress outwards, uniting their forces to give an increased charge in the carrier ball, at an increased distance from the source of power, and influencing each other so as to cause a second flexure in the contrary direction from the first one. All this appears to me to prove that the whole action is one of contiguous particles, related to each other, not merely in the lines which they may be conceived to form through the dielectric, between the inductric and the inducteous surfaces, but in other lateral directions also. It is this which gives the effect equivalent to lateral repulsion or expansion in the lines of force $I$ have spoken of, and enables induction to turn a corner (1304.). The power, instead of being like that of gravity, which relates particles together through straight lines, whatever other particles may be between them, is more analogous to that of a series of magnetic needles, or to the condition of the particles considered as forming the whole of a straight or a curved magnet. So that in whatever way $I$ view it, and with great suspicion of the influence of favourite notions over myself, I cannot perceive how the ordinary theory of induction can be a correct representation of that great natural principle of electrical action.

1232. I have had occasion in describing the precautions necessary in the use of the inductive apparatus, to refer to one founded on induction in curved lines (1203.); and after the experiments already described, it will easily be seen how great an influence the shell-lac stem may exert upon the 


\section{Mr. Faraday's Researches in Electricity. (Series XI.)}

charge of the carrier ball when applied to the apparatus (1218.), unless that precaution be attended to.

1233. I think it expedient, next in the course of these experimental researches, to describe some effects due to conduction, obtained with such bodies as glass, lac, sulphur, \&c., which had not been anticipated. Being understood, they will nlake us acquainted with certain precautions necessary in investigating the great question of specific inductive capacity.

1234. One of the inductive apparatus already described $(1187,8 \mathrm{c}$.) had a hemispherical cup of shell-lac introduced, which being in the interval between the inner ball and the lower hemisphere, nearly occupied the space there; consequently when the apparatus was charged, the lac was the dielectric or insulating medium through which the induction took place in that part. When this apparatus was first charged with electricity (1198.) up to a certain intensity, as $400^{\circ}$, measured by the Coulomb's electrometer (1180.), it sank much faster from that degree than if it had been previously charged to a higher point, and had gradually fallen to $400^{\circ}$; or than it would do if the charge were, by a second application, raised up again to $400^{\circ}$; all other things remaining the same. Again, if after having been charged for some time, as fifteen or twenty minutes, it was suddenly and perfectly discharged, even the stem having all electricity removed from it (1203.), then the apparatus being left to itself, would gradually recover a charge, which in nine or ten minutes would rise up to $50^{\circ}$ or $60^{\circ}$, and in one instance to $80^{\circ}$.

1235. The electricity which in these cases returned from an apparently latent to a sensible state, was always of the same kind as that which had been given by the charge. The return took place at both the inducing surfaces; for if after the perfect discharge of the apparatus the whole was insulated, as the inner ball resumed a positive state the outer sphere acquired a negative condition.

1236. This effect was at once distinguished from that produced by the exciter stem acting in curved lines of induction (1208. 1232.), by the circumstance that all the returned electricity could be perfectly and instantly discharged. It appeared to depend upon the shell-lac within, and to be, in some way, due to electricity evolved from it in consequence of a previous condition into which it had been brought by the charge of the metallic coatings or balls.

1237. To examine this state more accurately, the apparatus, with the hemispherical cup of shell-lac in it, was charged for about forty-five minutes to above $600^{\circ}$ with positive elec- 
tricity at the balls $h$ and B (fig. 1.) above and within. It was then discharged, opened, the shell-lac taken out, and its state examined; this was done by bringing the carrier ball near the shell-lac, uninsulating it, insulating it, and then observing what charge it had acquired. As it would be a charge by induction, the state of the ball would indicate the opposite state of electricity in that surface of the shell-lac which had produced it. At first the lac appeared quite free from any charge; but gradually its two surfaces assumed opposite states of electricity, the concave surface, which had been next the inner and positive ball, assuming a positive state, and the convex surface, which been in contact with the negative coating, acquiring a negative state; these states gradually increasing in intensity for some time.

1238. As the return action was evidently greatest instantly after the discharge, I again put the apparatus together, and charged it for fifteen minutes as before, the inner ball positively. I then discharged it, instantly removing the upper hemisphere with the interior ball, and, leaving the shelllac cup in the lower uninsulated hemisphere, examined its inner surface by the carrier ball as before (1237.). In this way I found the surface of the shell-lac actually negative, or in the reverse state to the ball which had been in it; this state quickly disappeared, and was succeeded by a positive condition, gradually increasing in intensity for some time, in the same manner as before. This first negative condition of the surface opposite the positive charging ball is a natural consequence of the state of things, the charging ball being in contact with the shell-lac only in a few points. It does not interfere with the general result and peculiar state now under consideration, except that it assists in illustrating in a very marked manner the ultimate assumption by the surfaces of the shell-lac of an electrified condition, similar to that of the metallic surfaces opposed to or against them.

1239. Glass was then examined with respect to its power of assuming this peculiar state. I had a thick flint glass hemispherical cup formed, which would fit easily into the space $o$ of the lower hemisphere (1188. 1189.); it had been heated and varnished with a solution of shell-lac in alcohol, for the purpose of destroying the conducting power of the vitreous surface. Being then well warmed and experimented with, I found it could also assume the same state, but not apparently to the same degree, the return action amounting in different cases to quantities from $6^{\circ}$ to $18^{\circ}$.

1240. Spermaceti experimented with in the same manner gave striking results. When the original charge had been 


\section{Mr. Faraday's Researches in Electricity. (Series XI.)}

sustained for fifteen or twenty minutes at about $500^{\circ}$, the return charge was equal to $95^{\circ}$ or $100^{\circ}$, and was about fourteen minutes arriving at the maximum effect. A charge continued for not more than two or three seconds was here succeeded by a return charge of $50^{\circ}$ or $60^{\circ}$. The observations formerly made (1234.) held good with this substance. Spermaceti, though it will insulate a low charge for some time, is a better conductor than shell-lac, glass, and sulphur; and this conducting power is connected with its readiness in exhibiting the particular effect under consideration.

1241. Sulphur.-I was anxious to obtain the amount of effect with this substance, first, because it is an excellent insulator, and in that respect would illustrate the relation of the effect to the degree of conducting power possessed by the dielectric (1247.); and in the next place, that I might obtain that body giving the smallest degree of the effect now under consideration, for the investigation of the question of specific inductive capacity (1277.).

1242. With a good hemispherical cup of sulphur cast solid and sound, I obtained the return charge, but only to an amount of $17^{\circ}$ or $18^{\circ}$. Thus glass and sulphur, which are bodily very bad conductors of electricity, and indeed almost perfect insulators, gave very little of this return charge.

1243. I tried the same experiment having air only in the inductive apparatus. After a continued high charge for some time I could obtain a little effect of return action, but it was ultimately traced to the shell-lac of the stem.

r244. I sought to produce something like this state with one electric power and without induction; for upon the theory of an electric fluid or fluids, that did not seem impossible, and then I should have obtained an absolute charge (1169. 1177.), or something equivalent to it. In this I could not succeed. I excited the outside of a cylinder of shell-lac very highly for some time, and then quickly discharging it (1203.), waited and watched whether any return charge would appear, but such was not the case. This is another fact in favour of the inseparability of the two electric forces, and another argument for the view that induction and its concomitant phænomena depend upon a polarity of the particles of matter.

1245. Although inclined at first to refer these effects to a peculiar masked condition of a certain portion of the forces, I think I have since correctly traced them to known principles of electrical action. 'The effects appear to be due to an actual penetration of the charge to some distance within the electric, at each of its two surfaces, by what we call conduction; so that, to use the ordinary phrase, the electric 
forces sustaining the induction are not upon the metallic surfaces only, but upon and within the dielectric also, extending to a greater or smaller depth from the metal linings. Let $c$ (fig. 10 .) be the section of a plate of any dielectric, $a$ and $b$ being the metallic coatings; let $b$ be uninsulated, and $a$ be charged positively; after ten or fifteen minutes, if $a$ and $b$ be discharged, insulated, and immediately examined, no electricity will appear in them; but in a short time, upon a second examination, they will appear charged in the same way, though not to the same degree, as they were at first. Now suppose that a portion of the positive force has, under the coercing influence of all the forces concerned, penetrated the dielectric and taken up its place at the line $p$, a corresponding portion of the negative force having also assumed its posi-

Fig. 10. tion at the line $n$; that in fact the electric at these two parts has become charged positive and negative; then it is clear that the induction of these two forces will be much greater one towards the other, and less in an external direction, now that they are at the small distance $n p$ from each other, than when they were at the larger interval $a b$. Then let $a$ and $b$ be discharged; the discharge destroys or neutralizes all external induction, and the coatings are therefore found by the carrier ball unelectrified; but it also removes almost the whole of the forces by which the electric charge was driven into the dielectric, and though probably a part goes forward in its passage and terminates in what we call discharge, the greater portion returns on its course to the surfaces of $c$, and consequently to the conductors $a$ and $b$, and constitutes the recharge observed.

1246. The following is the experiment on which I rest for the truth of this view. Two plates of spermaceti, $d$ and $f$ (fig. 11.), were put together to form the dielectric, $a$ and $b$ being the metallic coatings of this compound plate, as before. The system was charged, then discharged, insulated, examined, and found to give no indications of electricity to the carrier ball. The plates $d$ and $f$ were then separated from each other, and instantly $a$ with $d$ was found in a positive state, and $b$ with $f$ in a negative state, nearly all the electricity being in the linings $a$ and $b$. Hence it is clear that, of the forces sought for, the positive was in one half of the compound plate and the negative in the other half; for when removed bodily with the plates from each other's inductive in- 
fluence, they appeared in separate places, and resumed of necessity their power of acting by induction on the electricity of surrounding bodies. Had the effect depended upon a peculiar relation of the contiguous particles of matter only, then each half plate, $d$ and $f$, should have shown positive force on one surface and negative on the other.

1247. Thus it would appear that the best solid insulators, such as shell-lac, glass, and sulphur, have conductive properties to such an extent, that electricity can penetrate them bodily, though always subject to the overruling condition of induction (1178.). As to the depth to which the forces penetrate in this form of charge of the particles, theoretically, it should be throughout the mass, for what the charge of the metal does for the portion of dielectric next to it, should be done by the charged dielectric for the portion next beyond it again; but probably in the best insulators the sensible charge is to a very small depth only in the dielectric, for otherwise more would disappear in the first instance whilst the original charge is sustained, less time would be required for the assumption of the particular state, and more electricity would re-appear as return charge.

1248. The condition of time required for this penetration of the charge is important, both as respects the general relation of the cases to conduction, and also the removal of an objection that might otherwise properly be raised to certain results respecting specific inductive capacities, hereafter to be given (1269. 1277.).

1249. It is the assumption for a time of this charged state of the glass between the coatings in the Leyden jar, which gives origin to a well-known phænomenon, usually referred to the diffusion of electricity over the uncoated portion of the glass, namely, the residual charge. The extent of charge which can spontaneously be recovered by a large battery, after perfect uninsulation of both surfaces, is very considerable, and by far the largest portion of this is due to the return of electricity in the manner described. A plate of shell-lac six inches square, and half an inch thick, or a similar plate of spermaceti an inch thick, being coated on the sides with tinfoil as a Leyden arrangement, will show this effect exceedingly well.

1250. The peculiar condition of dielectrics which has now been described, is evidently capable of producing an effect interfering with the results and conclusions drawn from the use of the two inductive apparatus, when shell-lac, glass, \&c. are used in one or both of them (1192. 1207.); for upon dividing the charge in such cases according to the method described 
(1198. 1207.), it is evident that the one just receiving its half charge must fall faster in its tension than the other. For suppose app. i. first charged, and app. ii. used to divide with it; though both may actually lose alike, yet app. i., which has been diminished one half, will be sustained by a certain degree of return action or charge (1234.), whilst app. ii. will sink the more rapidly from the coming on of the particular state. I have endeavoured to avoid this interference by performing the whole process of comparison as quickly as possible, and taking the force of app. ii. immediately after the division, before any sensible diminution of the tension arising from the assumption of the peculiar state could be produced; and I have assumed that as about three minutes pass between the first charge of app. $i$. and the division, and three minutes between the division and discharge, when the force of the non-transferable electricity is measured, the contrary tendencies for those periods would keep that apparatus in a moderately steady and uniform condition for the latter portion of time.

1251. The particular action described occurs in the shelllac of the stems, as well as in the dielectric used within the apparatus. It therefore constitutes a cause by which the outside of the stems may in some operations become charged with electricity, independent of the action of dust or carrying particles (1203.).

[To be continued.]

XLIX. Justification of the Contact Theory of Galvanism. By G. Th. Fechner.

[Continued from p. 217.]

II. Facts which relate to the closed circuit.

1. T $\mathrm{T}$ appears according to the theory of contact as if iron, which when brought into contact with copper in water or diluted acids exhibits positive electricity, must on the contrary be positive in all other fluids; it acts however, nevertheless, negatively in a solution of sulphuret of potassium (Schrefelleber). Other examples nay be placed by the side of this, in which also the electromotive condition of the metals changes with the consistence of the intermediate fluid, for instance, that of tin and copper is in solution of ammonia the reverse of what it is in pure water, and that of copper and lead, in concentrated nitric acid, is the opposite of that in diJuted nitric acid, which immediately obviates the objection, 